\title{
Current Log-Periodic View on Future World Market Development
}

\author{
S. DrożD $\dot{Z}^{a, b, *}$,J. KWApień ${ }^{a}$, P. OśWiȩcimka ${ }^{a}$ And J. Speth ${ }^{a, c}$ \\ ${ }^{a}$ Institute of Nuclear Physics, Polish Academy of Sciences \\ Radzikowskiego 152, PL-31-342 Kraków, Poland \\ ${ }^{b}$ Institute of Physics, University of Rzeszów, PL-35-310 Rzeszów, Poland \\ ${ }^{c}$ Institut für Kernphysik, Forschungszentrum Jülich, D-52425 Jülich, Germany
}

Applicability of the concept of financial log-periodicity is discussed and encouragingly verified for various phases of the world stock markets development in the period 2000-2010. In particular, a speculative forecasting scenario designed in the end of 2004, that properly predicted the world stock market increases in 2007 , is updated by setting some more precise constraints on the time of duration of the present long-term equity market bullish phase. A termination of this phase is evaluated to occur in around November 2009. In particular, on the way towards this dead-line, a Spring-Summer 2008 increase is expected. On the precious metals market a forthcoming critical time signal is detected at the turn of March/April 2008 which marks a tendency for at least a serious correction to begin.

PACS numbers: 05.45.Pq, 52.35.Mw, 47.20.Ky

\section{Introduction}

The financial dynamics is a multiscale phenomenon and therefore the question which of its properties are scale invariant and which are scale characteristic refers to the essence of this phenomenon. There exists strong related evidence that at least a large portion of the financial dynamics is governed by phenomena analogous to criticality in the statistical physics sense [1]. In its conventional form criticality implies a scale invariance [2] of a properly defined function $\Phi(x)$ characterizing the system

$$
\Phi(\lambda x)=\gamma \Phi(x) .
$$

A constant $\gamma$ then describes how the properties of the system change under rescaling by the factor $\lambda$. The general solution to this equation reads [3]:

${ }^{*}$ corresponding author; e-mail: Stanislaw.Drozdz@ifj.edu.pl 


$$
\Phi(x)=x^{\alpha} \Pi(\ln (x) / \ln (\lambda)),
$$

where the first term represents a standard power-law that is characteristic of continuous scale-invariance with the critical exponent $\alpha=\ln (\gamma) / \ln (\lambda)$ and $\Pi$ denotes a periodic function of period one. This general solution can be interpreted in terms of discrete scale invariance. Due to the second term the conventional dominating scaling acquires a correction that is periodic in $\ln (x)$. Imprints of such oscillations can often be identified in the financial dynamics [4-7]. To make a proper mapping one defines $x=\left|T-T_{\mathrm{c}}\right|$, where $T$ denotes the clock time labelling the original price time series and represents a distance to the critical point $T_{\mathrm{c}}$. The emerging sequence of spacings between the corresponding consecutive repeatable structures at $x_{n}$ - as seen in the linear scale - forms a geometric progression according to the relation $\left(x_{n+1}-x_{n}\right) /\left(x_{n+2}-x_{n+1}\right)=\lambda$. The time points $T_{\mathrm{c}}$ thus correspond to the accumulation of such oscillations and, in the context of the financial dynamics such points mark a reversal of the trend. An important related element, for a proper interpretation and handling of the financial patterns as well as for consistency of the theory, is that such log-periodic oscillations manifest their action self-similarly through various timescales [7]. This applies both to the log-periodically accelerating bubble market phase as well as to the log-periodically decelerating anti-bubble phase.

Figure 1 schematically illustrates the relevant structures on one particular timescale. The thick solid line corresponds to the first term $\left(x^{\alpha}\right)$ in Eq. (2) and it represents the global market trends on both sides of $T_{\mathrm{c}}$, increasing and decreasing, respectively. On both these sides the log-periodic oscillations are superimposed, accelerating and decelerating, correspondingly. These oscillations are generated by the second term in Eq. (2). A specific form of the periodic function $\Pi$ in this equation is as yet not provided by any first principles. Since in the corresponding methodology the oscillation structure carries the most relevant information about the market dynamics, for transparency one uses the simplest representations for such a function. One reasonable possibility is the first term of its Fourier expansion,

$$
\Pi(\ln (x) / \ln (\lambda))=A+B \cos \left(\frac{\omega}{2 \pi} \ln (x)+\phi\right) .
$$

This implies that $\omega=2 \pi / \ln (\lambda)$. Already such a simple parameterization allows to properly reflect the contraction of oscillations, especially on the larger timescales. On the smaller timescales just replacing the cosine by its modulus equally well describes oscillations and in addition it often even better follows departures of the market amplitude from its average trend. Another simple - from the market perspective an even more realistic - representation of $\Pi$ is to use an asymmetric saw-like function. Such three possibilities are indicated in Fig. 1. What they all have however to obey is the same contraction ratio (preferred scaling factor) expressed by $\lambda_{1}$ and $\lambda_{2}$. For the real markets more and more evidence is collected that the preferred scaling factor $\lambda \approx 2$ and is common to all the scales and mar- 


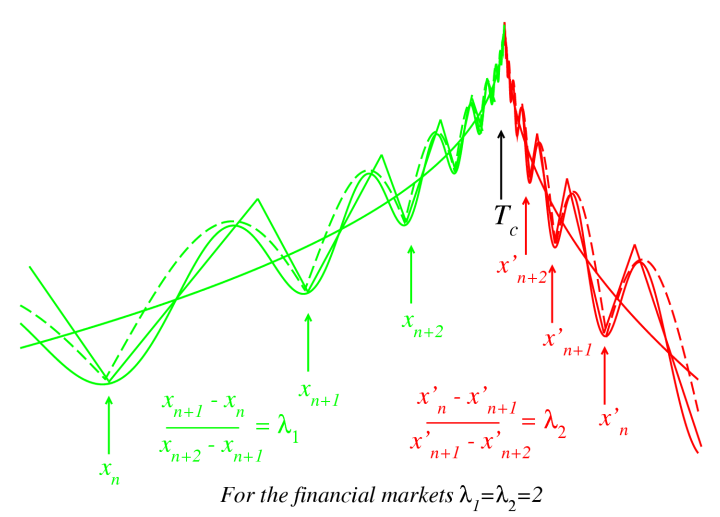

Fig. 1. Schematic illustration of the possible structures, relevant for the financial markets and consistent with Eq. (2). The thick - cusp-shaped at $T_{\mathrm{c}}$ - solid line corresponds to the conventional phase transition and in the financial context it reflects an overall market trend. Superimposed on top of this trend are three - cosine, cosine modulus and saw-like - possible practical representations for the oscillation pattern periodic in the logarithm of the distance $x$ from the critical time $T_{\mathrm{c}}\left(x=\left|T-T_{\mathrm{c}}\right|\right)$. Log-periodicity means that the ratios $\lambda_{1}$ and $\lambda_{2}$ of the distances between the consecutive repeatable structures $x_{n}$ are constant. Furthermore, for the financial markets $\lambda_{1}=\lambda_{2}=2$.

kets [8] both, in the log-periodically accelerating bubble and in the log-periodically decelerating anti-bubble phases [9]. We thus set $\lambda_{1}=\lambda_{2}=\lambda=2$. Universality of the $\lambda$, establishes very valuable and in fact crucial constraint on possible forms of the analytic representations of the market trends and of the oscillation patterns, including the future ones. This greatly amplifies a predictive power of the corresponding methodology. Also very helpful in this respect is the requirement of self-similarity which helps to clarify the significance of a given pattern and allows to determine on what timescale it operates. The present contribution provides further arguments in this favor.

\section{World stock market since 2003}

As far as the trends are concerned the contemporary stock market indices worldwide are remarkably synchronized. This is one of the characteristics reflecting the world globalization [10]. For the recession period since 2000 this effect is illustrated in Ref. [9]. This period ends approximately in the first months of 2003 and the stock markets synchronously enter the bullish period. As is shown in Fig. 2 all the indices assume the up trend. There of course are some differences in the magnitude of oscillation amplitudes but the common similarity of this oscillation pattern is clearly visible. Due to this similarity in the following we shall concentrate mostly on the American S\&P500 index because it represents the world largest market and is thus expected to provide the most reliable indicator of the global world market trends. 


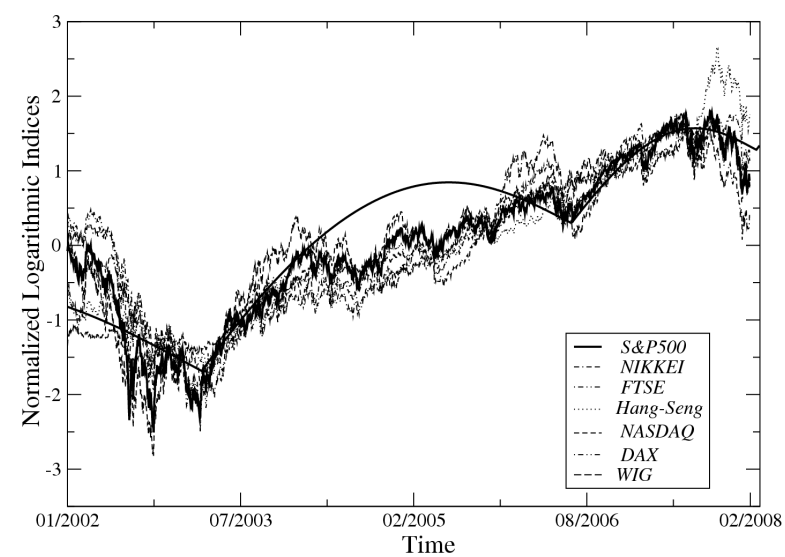

Fig. 2. Time series of the logarithm of the most important indices worldwide in the period 2002-2007. The time series have been appropriately normalized, $P(t) \rightarrow \frac{P(t)-\langle P(t)\rangle}{\sigma(P(t))}$, where $\langle\ldots\rangle$ denotes the average over the period under consideration and $\sigma$ is the standard deviation.
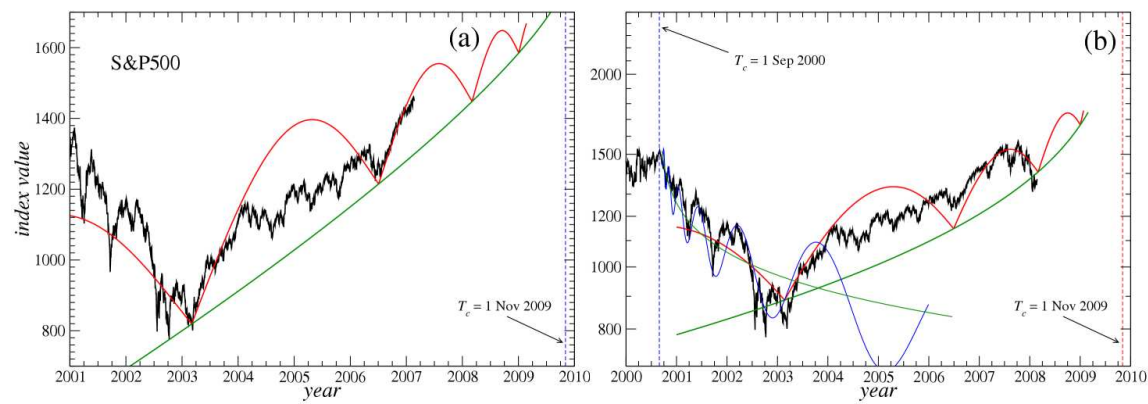

Fig. 3. (a) Time series of the S\&P500 from 1.1.2001 until 23.2.2007 versus the early (23.2.2007), presented at the FENS2007 Conference, optimal log-periodic representation (solid line) with $\lambda=2$ and $T_{\mathrm{c}}=1.11 .2009$ for the market bull phase. (b) The same log-periodic representation including the S\&P500 data up to 23.2.2008. Only the global trend is corrected as compared to (a). The dashed line indicates the log-periodically decelerating structure that started on 1.9.2000.

An early (made in February 2007, shown also at the time of FENS07 Conference) attempt to log-periodically grasp the large scale S\&P500 patterns and to provisionally estimate duration of its present increasing phase, before it enters recession of comparable magnitude as the one between mid 2000 and early 2003, is shown in Fig. 3. The corresponding critical time $T_{\mathrm{c}}$ points to the turn of October/November 2009. This scenario thus indicates that until this time the market, on average, should preserve its up trend. An extra argument in favor of this scenario is that it was predicting an intermediate sizeable correction in around the period November 2007- February 2008 and it took place indeed. It however 
also demands that in around the end of February 2008 (time of writing this contribution) this correction terminates and the index starts rising till at least late Summer before it starts correcting again. The reason for ignoring in this scenario the mid 2004 correction seen in the S\&P500 and comparable in magnitude to the one (relevant) in May-June 2006 is that this former correction is to be interpreted a remnant of the bear, since September 2000 log-periodically decelerating market component. The related part of this component is also drawn in the part (b) of Fig. 3. This part includes the S\&P500 data up to present and, accordingly, the global trend is updated, but the log-periodic structure remains unchanged.

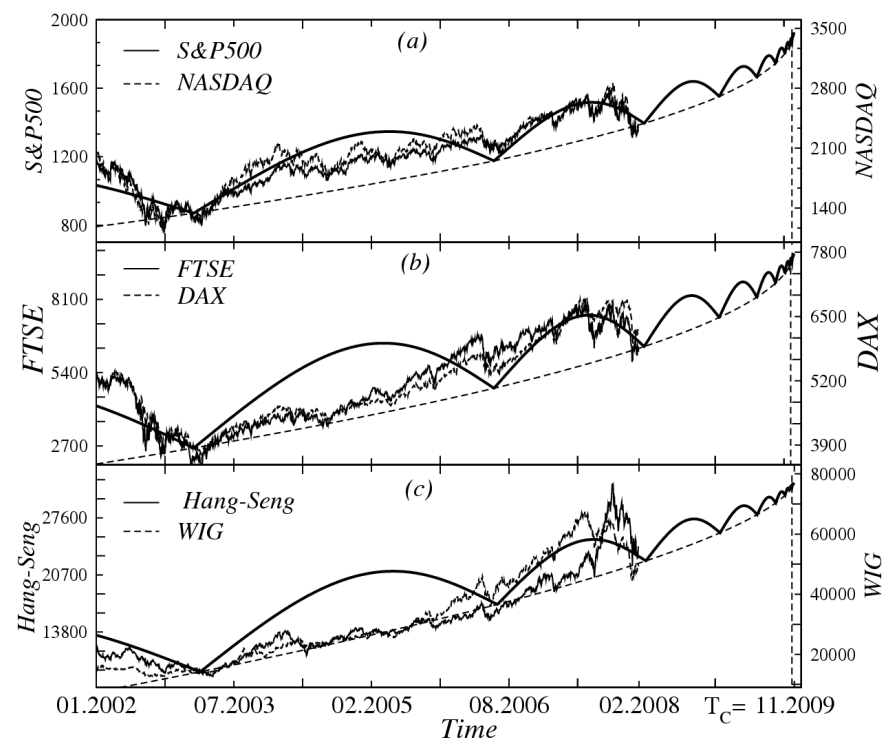

Fig. 4. (a) Time series from 1.1.2002 until 15.2.2008 for the three pairs of indices: (a) S\&P500 and NASDAQ, (b) DAX and FTSE and (c) HangSeng and WIG, versus the optimal log-periodic representation for the world bull market phase with $\lambda=2$ and $T_{\mathrm{c}}=1.11 .2009$.

The above updated scenario, including the real data up to present, for the three pairs of indices, S\&P500 and Nasdaq, DAX and FTSE, HangSeng and WIG (Poland), is shown in the three parts of Fig. 4. The log-periodic component remains here the same as the one in Fig. 3. The main reason for such a selection of indices presented in Fig. 4 is to show some from among the world most important ones whose oscillation patterns remain in a satisfactory agreement with the same common scenario, as well as those (HangSeng and WIG) whose blind relating to such a scenario may seem pointless. Let us recall here however a phenomenon of the "super-bubble" [8]. This is an effect that from time to time takes place in the financial dynamics and whose identification appears relevant for a proper interpretation of the financial patterns with the same universal value of the preferred 
scaling factor $\lambda=2$. This phenomenon of a "super-bubble" is a local boost, itself evolving log-periodically, superimposed on top of a long-term bubble and seen as an extra acceleration of the price increase. Such a "super-bubble" then crashes and the system returns to a normal bubble state that eventually crashes at the time determined by the long-term patterns. Two spectacular examples of such a phenomenon are provided by the Nasdaq in the first quarter of 2000 and by the gold price in the beginning of 1981 [8]. It seems very likely to us that the fast increases and then even faster decreases that we see in the last two indices of Fig. 4 during the period June 2006-February 2008 constitute further examples of the "super-bubbles" and the corresponding markets have just returned to their normal long-term (since March 2003) up trend and an ultimate critical time $T_{\mathrm{c}}$ may very well coincide with the same common scenario.

In Ref. [11] a speculative scenario for the stock market (S\&P500) development in the time period 2000-2010 was invented by representing the market index as a superposition of the two components: one declining and log-periodically decelerating since September 2000 and the second one rising and thus log-periodically accelerating. Based on the longest possible timescale (since 1800) log-periodic representation [8] for this index, the critical time for this second component was provisionally estimated in around September 2010. Time verified that so far this scenario makes a lot of sense. In particular, a spectacular increase in 2007 as well as the reverse of the increasing trend by the end of the year was predicted. Let us recall that this scenario was presented in November 2004. These facts encourage further its elaboration. Since, as discussed above, we now have a more precise estimate for the end of the present long-term bull market phase the scenario under consideration can be improved. An accordingly updated variant of this scenario

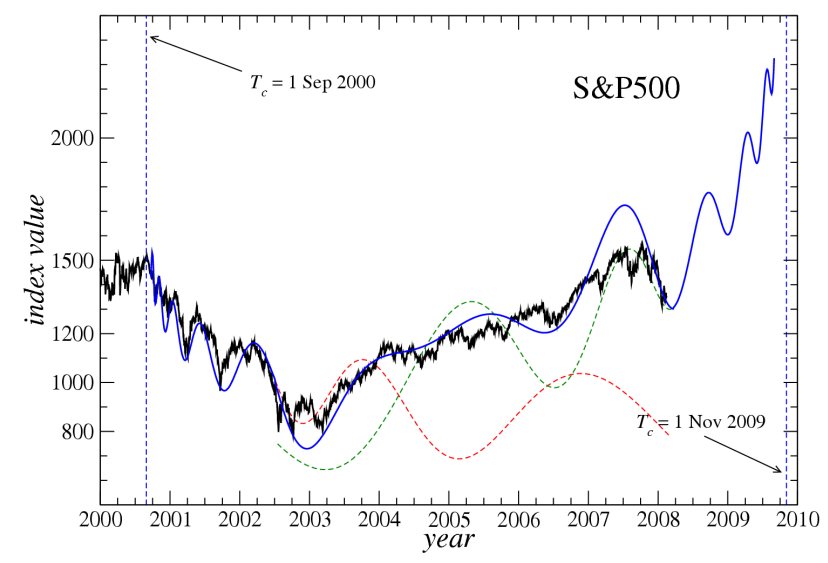

Fig. 5. A log-periodic scenario, represented by the solid line, for the S\&P500 development until end 2009. The critical time is fixed as $T_{\mathrm{c}}=1.11 .2009$, consistently with the signal from Fig. 3. This solid line is obtained by properly weighting and superimposing the two $\lambda=2$ components (dashed lines): log-periodically decelerating since 1.9.2000 and log-periodically accelerating towards 1.11 .2009 , correspondingly. 
is shown in Fig. 5. Interestingly, during the year 2007 this theoretical market representation opened room for an even stronger increase than what the S\&P500 has performed. Some other markets, like the ones shown in the part (c) of Fig. 4, made however use of this freedom and executed a more proportional detour.

\section{Precious metals market}

Some preliminary evidence already exists that the log-periodic oscillations may also accompany the commodities market dynamics during some more speculative periods. Such patterns have been identified on the gold market [8] during the time period 1978-1982, in this case including a very spectacular "super-bubble" before the ultimate reverse of the long-term trend, and on the oil futures market in the years 1998-2004 [11]. In this connection it needs to be also emphasized that the same preferred scaling factor $\lambda \approx 2$ as for the stock market turns out here appropriate.

After many years of stagnation the precious metals market revitalized starting approximately in the second half of the 2000-2003 stock market recession period. The following development, at least partly driven by speculative activity, elevated the precious metals prices in a relatively short period of time to the level a factor of 3 to 4 higher. This is illustrated in Fig. 6 which shows the gold, the

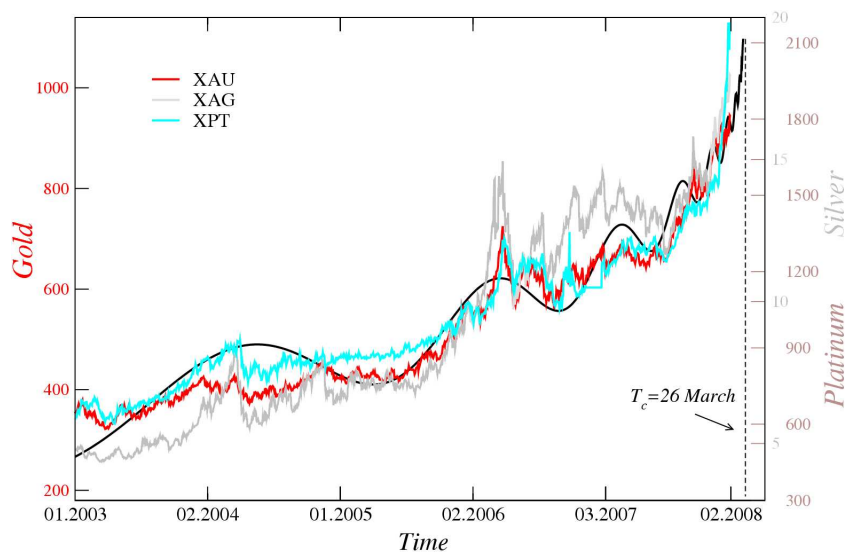

Fig. 6. Time series of the gold XAU, silver XAG and platinum XPT prices (in USD) since 1.1.2003 until 22.2.2008, versus their optimal log-periodic representation with $\lambda=$ 2. The corresponding critical time $T_{\mathrm{c}}=26.3 .2008$.

silver and the platinum price changes during the period January 2003-February 2008. The oscillation patterns of all these prices resemble each other and, in addition, they quite convincingly coincide with the log-periodic $(\lambda=2)$ structure which points to the turn of March/April 2008 as the critical time $T_{\mathrm{c}}$. Whether this indicates an ultimate reversal of the precious metals present price trends in around this time or only a sizeable (20-30\%) correction cannot be settled at this 
stage of the development. If the later possibility is to occur, i.e., after such a correction the market starts returning to the previous highs, then - in the spirit of self-similar log-periodicity [7] — it is expected to assume increase towards significantly higher levels and this market phase should last for another 3-4 years in order to complete the full log-periodic cycle on the appropriately longer time horizon, such that the whole log-periodic structure seen in Fig. 6 constitutes its self-similarly nested substructure. It seems also likely that the last few months accelerated increases, especially on the platinum market, can be interpreted in terms of a "super-bubble". In any case however at the $T_{\mathrm{c}}$ indicated above one may expect the beginning of a significant correction on the precious metals market.

\section{Acknowledgments}

J.S. thanks the Foundation for Polish Science for financial support through the Alexander von Humboldt Honorary Research Fellowship.

\section{References}

[1] D. Sornette, Why Stock Markets Crash: Critical Events in Complex Financial Systems, Princeton University Press, Princeton 2003.

[2] H.E. Stanley, Introduction to Phase Transitions and Critical Phenomena, Oxford University Press, New York 1971.

[3] J. Nauenberg, J. Phys. A 8, 925 (1975); G. Jona-Lasinio, Nuovo Cimento 26B, 99 (1975).

[4] D. Sornette, A. Johansen, J.-P. Bouchaud, J. Phys. I (France) 6, 167 (1996).

[5] J.A. Feigenbaum, P.G.O. Freund, Int. J. Mod. Phys. B 10, 3737 (1996).

[6] N. Vandevalle, M. Ausloos, Ph. Boveroux, A. Minguet, Eur. Phys. J. B 4, 139 (1998).

[7] S. Drożdż, F. Ruf, J. Speth, M. Wójcik, Eur. Phys. J. B 10, 589 (1999).

[8] S. Drożdż, F. Grümmer, F. Ruf, J. Speth, Physica A 324, 174 (2003).

[9] M. Bartolozzi, S. Drożdż, D.B. Leinweber, J. Speth, A.W. Thomas, Int. J. Mod. Phys. C 16, 1347 (2005).

[10] S. Drożdż, F. Grümmer, F. Ruf, J. Speth, Physica A 294, 226 (2001).

[11] S. Drożdż, F. Grümmer, F. Ruf, J. Speth, in: Practical Fruits of Econophysics: Proceedings of the Third (2004) Nikkei Econophysics Symposium, Ed. H. Takayasu, Springer, Tokyo 2006, p. 93; available also as arXiv:physics/0503006. 